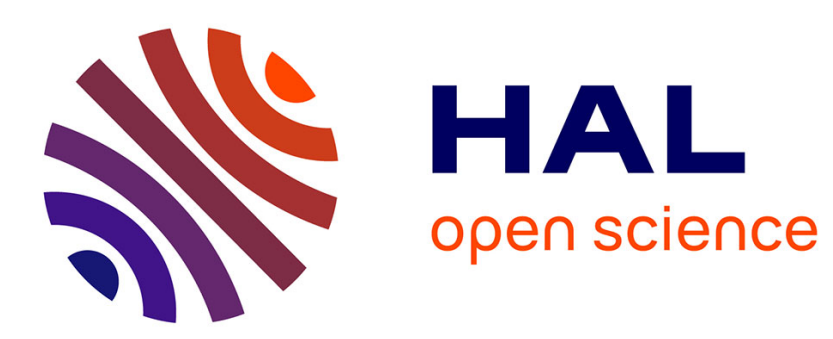

\title{
LES SAVOIRS DANS LE CHAMP DE L'ACCUEIL DES ENFANTS
}

\author{
Nicolas Murcier
}

\section{To cite this version:}

Nicolas Murcier. LES SAVOIRS DANS LE CHAMP DE L'ACCUEIL DES ENFANTS. Informations sociales, 2006. hal-01998847

\section{HAL Id: hal-01998847 \\ https://hal.science/hal-01998847}

Submitted on 13 Sep 2019

HAL is a multi-disciplinary open access archive for the deposit and dissemination of scientific research documents, whether they are published or not. The documents may come from teaching and research institutions in France or abroad, or from public or private research centers.
L'archive ouverte pluridisciplinaire HAL, est destinée au dépôt et à la diffusion de documents scientifiques de niveau recherche, publiés ou non, émanant des établissements d'enseignement et de recherche français ou étrangers, des laboratoires publics ou privés. 


\section{LES SAVOIRS DANS LE CHAMP DE L'ACCUEIL DES ENFANTS Idéologies, mots d'ordre, modes \\ Nicolas Murcier}

Caisse nationale d'allocations familiales (CNAF) | «Informations sociales »

$2006 / 5 n^{\circ} 133$ | pages 30 à 37

ISSN 0046-9459

Article disponible en ligne à l'adresse :

https://www.cairn.info/revue-informations-sociales-2006-5-page-30.htm

\section{Pour citer cet article :}

Nicolas Murcier, «Les savoirs dans le champ de l'accueil des enfants. Idéologies, mots d'ordre, modes », Informations sociales 2006/5 ( $\mathrm{n}^{\circ} 133$ ), p. 30-37.

Distribution électronique Cairn.info pour Caisse nationale d'allocations familiales (CNAF).

(C) Caisse nationale d'allocations familiales (CNAF). Tous droits réservés pour tous pays.

La reproduction ou représentation de cet article, notamment par photocopie, n'est autorisée que dans les limites des conditions générales d'utilisation du site ou, le cas échéant, des conditions générales de la licence souscrite par votre établissement. Toute autre reproduction ou représentation, en tout ou partie, sous quelque forme et de quelque manière que ce soit, est interdite sauf accord préalable et écrit de l'éditeur, en dehors des cas prévus par la législation en vigueur en France. Il est précisé que son stockage dans une base de données est également interdit. 


\section{Les savoirs dans le champ de l'accueil des enfants}

\section{Idéologies, mots d'ordre, modes}

Sur quels savoirs s'appuie-t-on, depuis un siècle, pour organiser l'accueil des enfants et les relations avec les familles? Les préoccupations hygiénistes ont progressivement laissé la place à une centration sur le développement affectif et la relation mère/enfant. D'où l'accent mis sur le repérage des dysfonctionnements dans la relation parent/enfant, et surtout mère/enfant. Cette primauté affichée du rôle maternel a participé (et participe) à l'assignation aux femmes de pourvoir de façon quasi exclusive aux soins et à l'éducation des jeunes enfants.

Si la garde des bébés et des jeunes enfants a toujours été une préoccupation dépendant des contextes socio-historiques, des cultures, des connaissances et des représentations sociales de l'enfance, l'accueil collectif de jeunes enfants a été, dès son origine, objet de controverses et de débats. Cet article propose de repérer certains mots d'ordre, idéologies et modes qui se sont développés au cours du $\mathrm{XX}^{\mathrm{e}}$ siècle au sein des modes de garde pour jeunes enfants, et qui sont actuellement à l'œuvre.

\section{Suspicion de l'accueil collectif}

$\mathrm{Au}$ cours du $\mathrm{XX}^{\mathrm{e}}$ siècle, les institutions proposant un accueil collectif sont suspectées de favoriser des carences affectives et deviennent dès lors l'objet d'une polémique sur les effets pathogènes de la collectivité. La séparation mère/enfant sera stigmatisée comme expliquant les relations carencées et l'apparition de troubles du comportement et/ou du développement chez les tout-petits, sans que soient réellement interrogées les conditions de l'accueil : les sureffectifs, la formation 
(ou la non-formation dans un grand nombre de cas) des professionnelles, le manque de contact individualisé, le non-accueil des parents dans les établissements, l'aménagement de la séparation entre l'enfant et l'adulte tutélaire, etc. (Bouve, 2001 ; Neyrand, 2000). La mise en avant des effets nocifs de la crèche a constitué une exagération abusive. En effet, les différents travaux (notamment ceux de Spitz) menés alors ont porté sur des institutions particulières (pouponnières, hôpitaux) dans lesquelles les enfants vivaient en permanence. La généralisation des effets produits par ces institutions à toutes celles prenant en charge des tout-petits, dont les crèches collectives, n'avait-elle pas pour objectif de permettre "au modèle idéologique de la femme au foyer qui élève ses enfants - défendu par le courant familialiste ou "néo-spitzien "-de s'affirmer de nouveau tout en renouvelant le système d'opposition aux crèches : la crèche contribuerait toujours à la mortalité infantile, si ce n'est plus par son incompétence hygiénique et médicale, c'est alors par son insuffisance affective, sa pauvreté psychique, les actes mécanisés et dépersonnalisants du personnel" (Bouve, 2001) ?

\section{L'ouverture des établissements aux familles}

En 1975, les parents sont officiellement admis à entrer dans les crèches. À partir de ces années, les objectifs de ces dernières sont redéfinis. Il s'agit maintenant pour elles de participer à la prévention de la santé psychique et mentale, à l'éducation et à l'éveil des jeunes enfants. Le décret $\mathrm{n}^{\circ} 2000-762 \mathrm{du} 1^{\text {er }}$ août 2000, relatif aux établissements et services d'accueil des enfants de moins de 6 ans, leur donne comme missions de participer au soutien de la fonction parentale, de permettre la conciliation entre vie familiale et vie professionnelle et d'accueillir les jeunes enfants en situation de handicap ou de maladie chronique (Art. r. 180-1). La place des parents au sein de l'institution et leur participation à la vie de l'établissement ou du service doivent dorénavant être définies (Art. r. 180-10). Cependant, cette définition ne constitue pas en soi une nouveauté, car sous le ministère de Georgina Dufoix, il en était déjà question, notamment par la mise en place de conseils de crèche. 
La circulaire 83/22 du 30 juin 1983, relative à la participation des parents à la vie quotidienne de la crèche, recommandait la mise en place d'un conseil de crèche au sein de chaque établissement. Actuellement, relativement peu d'établissements ont effectivement institué une telle instance, ce qui met en évidence leur difficulté à travailler avec les parents (Ott, 2004).

\section{La centration sur le développement affectif}

Après la Seconde Guerre mondiale, les médecins découvrent l'affectivité et son importance dans le développement du bébé et du jeune enfant. Les recherches se multiplient donc particulièrement dans le champ de la psychanalyse, de la pédopsychiatrie et de la psychologie pour tendre vers une compréhension des troubles du jeune enfant. Les psychanalystes (entre autres, Bowlby, Dolto, Spitz et Winnicott) mettent alors en avant la primauté de la mère, l'importance fondamentale de la relation mère/enfant dans le développement du jeune enfant, introduisant une hiérarchie dans l'ordre des besoins (Sellenet, 2005). La mère devient dès lors incontournable. De cette construction sociale est née la sur-responsabilisation des mères et la déresponsabilisation des pères (Castelain-Meunier, 2002), toujours actuelles, plaçant en permanence les mères sous le regard des professionnel(le)s de la petite enfance et/ou de la protection de l'enfance. En effet, dès que la relation mère/enfant n'est pas "suffisamment bonne", qu'elle est contrariée par des obstacles, ou qu'est abordée la séparation mère/enfant, les professionnel(le)s se trouvent irrésistiblement ramené(e)s à la théorie de l'attachement et à son cadre de référence (Le Capitaine et al., 2002 ; Murcier, 2005 ; Sellenet, 2005). Ceux-ci ou celles-ci étant conforté(e)s par "les discours de nombre d'experts de la petite enfance [...] qui contribuent à légitimer "scientifiquement » l'argument selon lequel le jeune enfant a, avant tout, besoin de sa mère" (Fagnani, 2000).

Jusqu'à la fin des années soixante-dix, la question de la présence du père auprès du jeune enfant ne se pose pas. Il faut attendre le début des années quatre-vingt pour que les recherches commencent à s'intéresser à son rôle et à sa présence auprès du tout-petit (Le Camus, 2000 ; 
Neyrand, 2000). Si le père doit avoir une action, celleci interviendra ultérieurement, notamment lorsque l'enfant aura quitté la prime enfance et atteint l'âge de la marche (Dolto, 1988). Durant longtemps, il a donc été exclu des soins physiques et psychiques du bébé et du jeune enfant. Son rôle est décrit comme très différent de celui de la mère, et se cantonne essentiellement à la fonction d'autorité (Le Camus, 2000). Jusqu'aux années soixante-quinze, des facteurs économiques participent à cette vision et ségrégation des rôles maternels et paternels. En effet, durant cette période, l'activité féminine à l'extérieur de la famille est encore réduite même si le travail des femmes a toujours existé (Schweitzer, 2002), selon des modalités différentes en fonction du contexte socio-historique, des cultures et du système de production d'une société donnée -, comme est réduite la présence du père dans la cellule familiale au cours de la journée dans cette période de pleinemploi. Actuellement, la part des femmes dans le monde du travail représente plus de $45 \%$. En 2002, le taux d'activité de celles âgées de 25 à 49 ans est de 81,3\% (INSEE, 2004). Une majorité d'entre elles travaillent donc aujourd'hui à l'extérieur, comme des pères sont présents au sein du foyer lors de la journée (pères chômeurs, RMistes, en congé parental, etc.). Pourtant, les discours psychologiques et les idéologies existants avant les mutations dans le monde du travail demeurent largement.

\section{Une focalisation sur la prévention des relations pathogènes mère/enfant}

Les professionnelles de la petite enfance se sont donc trouvées légitimées en tant que substituts maternels devant limiter l'apparition ou l'installation de carences liées à la séparation du bébé d'avec sa mère, en assurant une continuité des soins maternels. L'inscription dans le champ paramédical de ces professions a d'ailleurs concouru - et concourt toujours - à une vigilance accrue envers le "pathologique" au détriment du "normal", y compris dans les institutions d'accueil de la petite enfance. Formées au soin, leur regard se centre sur ce qui dysfonctionnerait, notamment dans la relation mère/enfant. Ainsi, selon une auxiliaire de puéri- 
culture rencontrée, "très tôt, l'enfant manifeste son mal-être, et c'est [aux] professionnelles de la petite enfance d'essayer de pallier cette souffrance. Déjà dans les structures d'accueil, il faut observer la relation mèrelenfant pour voir si tout se passe bien, mettre en place un travail de collaboration avec divers partenaires, aider ces tout jeunes enfants à communiquer autrement que par la violence et accompagner la relation mère/enfant lorsque que celle-là ne marche pas bien".

\section{L'imposition du modèle de parentalité de la classe moyenne}

La démarche professionnelle des accueillantes, dont une grande majorité a reçu une formation paramédicale, s'étaye largement sur leur expérience propre, notamment sur leur expérience maternelle et/ou sur l'imago maternelle. Le regard curatif déployé par les professionnelles et la matri-focalisation des représentations ont pour corollaire une recherche de comportements pseudo pathologiques - c'est-à-dire que les professionnelles se trouvent dans la suspicion vis-à-vis des comportements parentaux, essentiellement maternels, qui ne rentrent pas dans le cadre de leurs représentations impliquant un contrôle de la relation mère/enfant (Donzelot, 1977 ; Ott, 2004).

Les institutions d'accueil de la petite enfance ont également pour mission de prévenir, de repérer et de signaler tout signe de carence, de mauvais traitement et d'abus de la part des parents. En effet, le jeune enfant, et plus généralement l'enfant, est actuellement un individu à protéger, de lui-même mais également et surtout des autres, notamment de ses parents (ou de ses éducateurs), potentiellement maltraitants ou incestueux, etc. (Gavarini et Petitot, 1998). Aujourd'hui, venant se juxtaposer à la figure de l'enfant en danger, une autre se dessine : celle de l'enfant "dangereux" dont la société cherche à se protéger, le même enfant pouvant être, tour à tour ou simultanément, enfant en danger et/ou enfant dangereux. Ainsi, l'obsession actuelle de la délinquance donne lieu à des recommandations de prévention à des âges de plus en plus bas (dès la crèche, selon l'INSERM (2005) et le rapport Bénisti). Il reviendrait 
aux professionnel(le)s de la petite enfance de repérer précocement les attitudes qui pourraient caractériser le trouble des conduites et les comportements délictueux à venir, mais également de suivre les familles dites "à risques" (INSERM, 2005).

\section{Sur quels savoirs les professionnel(le)s prennent-ils appui ?}

Certains concepts psychanalytiques ont été largement investis par les professionnel(le)s de la petite enfance. Cependant, l'utilisation qui en est faite apparaît très éloignée de l'élaboration théorique des différents auteurs. Les sociologues Thierry Blöss et Sophie Odena (2005) montrent ainsi l'utilisation abusive que certains professionnel(le)s font par exemple du concept d'“objet transitionnel'. Celui-ci s'est banalisé dans les institutions d'accueil, et est le plus souvent dénommé par le vocable "doudou". Les mêmes sociologues analysent la façon dont l'utilisation d'arguments psychologiques ou médicaux permet aux professionnel(le)s d'imposer aux parents certaines idéologies. L'emploi du terme "doudou", massivement présent dans les discours, signifie en permanence aux mères combien leur absence est difficile pour leur enfant, véhiculant également que le très jeune enfant ne peut être bien qu'en leur présence. Ce vocable semble être devenu un générique, un mot standardisé qui rappelle aux mères que c'est à elles que revient de pourvoir, de façon quasi exclusive, aux besoins de leur(s) enfant(s).

Les idéologies de la primauté maternelle, de la dyade mère/enfant et de la répartition des rôles parentaux qui se sont développées au cours du $\mathrm{XX}^{\mathrm{e}}$ siècle sont encore largement présentes et véhiculées par les centres de formation aux différents métiers de la petite enfance. Nous entendons par idéologies des élaborations théoriques "qui constituent aujourd'hui des sortes de dogmes, des croyances imposées comme des vérités incontournables" (Sellenet, 2005). Les théories psychologiques sont des constructions sociales qu'il convient de déconstruire. Catherine Sellenet (2005) montre comment certaines théories dogmatiques, notamment celle de la primauté de la mère, sont toujours mises en avant, alors que les observations ayant 


\section{Bibliographie}

> T. Blöss, S. Odena, "Idéologies et pratiques sexuées des rôles parentaux. Quand les institutions de garde des jeunes enfants en confortent le partage inégal", Recherches et prévisions, $n^{\circ} 80$, juin 2005, p. 77-91.

$>C$. Bouve, Les crèches collectives : usagers et représentations sociales, Paris, L'Harmattan, 2001.

$>$ D. Boyer, S. Renouard, "Les hommes bénéficiaires de I'APE. Quels arbitrages au sein des couples ?", Dossiers d'études, $n^{\circ}$ 57, juin 2004.

$>$ C. Castelain-Meunier, La place des hommes et les métamorphoses de la famille, Paris, PUF, 2002.

> D.-L. Chaplain, M.-F. CustosLucidi, Les métiers de la petite enfance. Des professions en quête d'identité, Paris, Syros, 2001.

> F. Dolto, Quand les parents se séparent, Paris, Le Seuil, 1988.

> J. Donzelot, La police des familles, Paris, Éditions de Minuit, 1977.

$>$ J. Fagnani, Un travail et des enfants. Petits arbitrages et grands dilemmes, Paris, Bayard, 2000.

$>$ L. Gavarini, F. Petitot, La fabrique de I'enfant maltraité. Un nouveau regard sur l'enfant et la famille, Ramonville Saint-Agne, Érès, 1998.

$>$ S. Giampino, Les mères qui travaillent sont-elles coupables?, Paris, Albin Michel, 2000.

$>$ INSEE, Femmes et hommes. Regards sur la parité, Paris, 2004.

> INSERM, Troubles des conduites chez l'enfant et l'adolescent, Paris, 2005. permis de les élaborer étaient "d'emblée biaisées par le contexte d'observation". Actuellement, davantage de pères s'impliquent et fréquentent le mode de garde, même si statistiquement les mères assument davantage cette tâche et si l'implication de ces derniers dépend de différents facteurs, notamment de la disponibilité (horaires atypiques, aménagement du temps de travail, emploi moins qualifié que la mère, etc.) (Boyer, Renouard, 2004). L'existence d'une crèche d'entreprise sur le lieu de travail est également un facteur déterminant pour l'investissement des pères. Cependant, ceuxci restent peu accueillis par les professionnelles de la petite enfance : "Les pères, on ne les voit jamais ou peu. Mais quand ils s'impliquent, il y a toujours ce rappel à la mère : "Vous direz à votre femme que... », comme s'ils n'étaient pas des interlocuteurs à part entière, ceux à qui les professionnelles désirent s'adresser" ; "Lors des transmissions, c'est vrai que les professionnelles s'adressent toujours aux mères, même quand c'est le père qui vient chercher son enfant", témoignent des éducatrices de jeunes enfants. Ainsi, nombre de professionnelles disqualifient les pères au sein des institutions et survalorisent les mères (Blöss et Odena, 2005 ; Chaplain et Custos-Lucidi, 2001). Ce fait est à mettre en corrélation avec la formation reçue par les professionnelles et sa centration sur la relation mère/enfant (Murcier, 2006). Comme le souligne Marie-Dominique Wilpert (2005), dans les discours est présent un paradoxe "au fondement de la pensée et des savoirs concernant les modes de garde des jeunes enfants [fragilisant] la légitimité des modes de garde [et assignant] fantasmati[quement] ces lieux à une place de dépôt, de consigne de bébés qui attendent leur véritable destination : leur mère". Selon l'analyse de Thierry Blöss et Sophie Odena (2005), les institutions de garde pour enfants de moins de 6 ans jouent un rôle dans la perpétuation du partage inégal des pratiques parentales comme de l'idéologie que la responsabilité des jeunes enfants concerne, avant tout, les mères. En faisant appel en permanence aux mères et en survalorisant le rôle maternel, les institutions d'accueil de la petite enfance contribuent, pas forcément de façon consciente, à la reproduction de la division sexuelle des rôles parentaux. 


\section{Une double contrainte}

Il semble aujourd'hui que les parents soient soumis à une double contrainte : d'un côté, il leur est signifié, surtout aux mères, qu'ils sont indispensables pour leur(s) enfant(s) (Ott, 2004) ; de l'autre, implicitement ou explicitement, il leur est dit : "Vous ne savez pas faire". L'enfant est renvoyé à sa famille, mais les différentes institutions d'accueil, et en leur sein les professionnel(le)s, contrôlent, surveillent la façon dont les parents s'en occupent. Dans ce contexte, une double relation de suspicion s'établit. D'une part, une suspicion de non-qualité de la prestation d'accueil de la part des parents, actuellement reconnus comme "clients" par les professionnel(le)s ; et d'autre part, une suspicion de non-compétence de la part des professionnel(le)s, cette fois-ci non seulement vis-à-vis des mères, mais également vis-à-vis du milieu familial. C'est-à-dire que les professionnel(le)s de la petite enfance sont certainement à la jonction de deux dangers : premièrement, une logique marchande de production de service qui ferait des parents leurs "clients"; deuxièmement, une logique de police des familles qui les pousserait à leur imposer des idéologies et des "bonnes" pratiques. Sans doute, ces deux dangers ne s'excluent-ils pas l'un l'autre. La "marchandisation" vise davantage les parents aisés (les établissements et les services d'accueil privés sont actuellement en plein essor), et la police des familles, les autres, notamment les familles catégorisées comme étant "à risques" (selon l'INSERM). L'accueil des jeunes enfants au sein des modes de garde et le développement des places offertes constituent une nécessité pour tendre vers l'égalité entre les femmes et les hommes. Cela implique également que les parents disposent d'un véritable choix du mode d'accueil et que ne leur soit par perpétuellement renvoyée une image négative, par exemple lorsque ceux qui bénéficient d'une place en crèche confient leur enfant pour une durée journalière comprise entre dix et douze heures.
> J. Le Camus, Le vrai rôle du père, Paris, Odile Jacob, 2000.

> B. Le Capitaine et al., Guide de l'éducateur de jeunes enfants, Paris, Dunod, 2002.

$>$ N. Murcier, "Petite enfance et rapports sociaux de sexe : la formation des professionnel(le)s de la petite enfance, idéologies et représentations sociales", in Y. GuichardClaudic, D. Kergoat et A. Vilbrod (coord.), L'inversion du genre. Quand les métiers masculins se conjuguent au féminin et réciproquement, actes du colloque de Brest, AFS, à paraître, 2006.

$>$ N. Murcier, "Le loup dans la bergerie. Prime éducation et rapports sociaux de sexe", Recherches et prévisions, $n^{\circ}$ 80, juin 2005, p. 67-75. > G. Neyrand, L'enfant, la mère et la question du père. Un bilan critique de l'évolution des savoirs sur la petite enfance, Paris, PUF, 2000.

$>$ L. Ott, Travailler avec les familles, Ramonville Saint-Agne, Érès, 2004. $>$ S. Schweitzer, Les femmes ont toujours travaillé : une histoire du travail des femmes aux XIX ${ }^{e}$ et XX $X^{e}$ siècles, Paris, Odile Jacob, 2002.

$>$ C. Sellenet, Les pères vont bien ! Comment les hommes affirment et assument aujourd'hui leur paternité, Paris, Flammarion, 2005.

> R.-A. Spitz, De la naissance à la parole, Paris, PUF, 1968.

> M.-D. Wilpert, "Bonne mère... au foyer", La lettre de l'enfance et de I'adolescence, $n^{\circ}$ 59, mars 2005, p. 35-41. 